PROCEEDINGS OF THE

AMERICAN MATHEMATICAL SOCIETY

Volume 131, Number 12, Pages 3851-3859

S 0002-9939(03)07083-7

Article electronically published on May 5, 2003

\title{
FIXED POINTS IN THE FAMILY OF CONVEX REPRESENTATIONS OF A MAXIMAL MONOTONE OPERATOR
}

\author{
B. F. SVAITER
}

(Communicated by Jonathan M. Borwein)

\begin{abstract}
Any maximal monotone operator can be characterized by a convex function. The family of such convex functions is invariant under a transformation connected with the Fenchel-Legendre conjugation. We prove that there exists a convex representation of the operator which is a fixed point of this conjugation.
\end{abstract}

\section{INTRODUCTION}

Let $X$ be a real Banach space and $X^{*}$ its dual. It is usual to identify a point to set operator $T: X \rightrightarrows X^{*}$ with its graph, $\left\{\left(x, x^{*}\right) \in X \times X^{*} \mid x^{*} \in T(x)\right\}$. We will use the notation $\left\langle x, x^{*}\right\rangle$ for the duality product $x^{*}(x)$ of $x \in X, x^{*} \in X^{*}$.

An operator $T: X \rightrightarrows X^{*}$ is monotone if

$$
\left(x, x^{*}\right),\left(y, y^{*}\right) \in T \Rightarrow\left\langle x-y, x^{*}-y^{*}\right\rangle \geq 0
$$

and is maximal monotone if it is monotone and

$$
\forall\left(y, y^{*}\right) \in T,\left\langle x-y, x^{*}-y^{*}\right\rangle \geq 0 \Rightarrow\left(x, x^{*}\right) \in T .
$$

Krauss 11 managed to represent maximal monotone operators by subdifferentials of saddle functions on $X \times X$. After that, Fitzpatrick [8] proved that maximal monotone operators can be represented by convex functions on $X \times X^{*}$. Later on, Simons [19] studied maximal monotone operators using a min-max approach. Recently, the convex representation of maximal monotone operators was rediscovered by Burachik and Svaiter [7] and Martinez-Legaz and Théra [13. In [7, some results on enlargements are used to perform a systematic study of the family of convex functions which represents a given maximal monotone operator. Here we are concerned with this kind of representation.

Given $f: X \rightarrow \overline{\mathbb{R}}$, the Fenchel-Legendre conjugate of $f$ is $f^{*}: X^{*} \rightarrow \overline{\mathbb{R}}$,

$$
f^{*}\left(x^{*}\right):=\sup _{x \in x}\left\langle x, x^{*}\right\rangle-f(x) .
$$

Received by the editors July 31, 2002.

2000 Mathematics Subject Classification. Primary $47 \mathrm{H} 05$.

Key words and phrases. Maximal monotone operators, conjugation, convex functions.

This work was partially supported by CNPq Grant 301200/93-9(RN) and by PRONEXOptimization. 
The subdifferential of $f$ is the operator $\partial f: X \rightrightarrows X^{*}$,

$$
\partial f(x):=\left\{x^{*} \in X^{*} \mid f(y) \geq f(x)+\left\langle y-x, x^{*}\right\rangle, \forall y \in X\right\} .
$$

If $f$ is convex, lower semicontinuous and proper, then $\partial f$ is maximal monotone [17]. From the previous definitions, we have the Fenchel-Young inequality: for all $x \in X$, $x^{*} \in X^{*}$,

$$
f(x)+f^{*}\left(x^{*}\right) \geq\left\langle x, x^{*}\right\rangle, f(x)+f^{*}\left(x^{*}\right)=\left\langle x, x^{*}\right\rangle \Longleftrightarrow x^{*} \in \partial f(x) .
$$

So, defining $h_{\mathrm{FY}}: X \times X^{*} \rightarrow \overline{\mathbb{R}}$,

$$
h_{\mathrm{FY}}\left(x, x^{*}\right):=f(x)+f^{*}\left(x^{*}\right),
$$

we observe that this function fully characterizes $\partial f$. Assume that $f$ is convex, lower semicontinuous and proper. In this case, $\partial f$ is maximal monotone. Moreover, if we use the canonical injection of $X$ in to $X^{* *}$, then $f^{* *}(x)=f(x)$ for all $x \in X$. Hence, for all $\left(x, x^{*}\right) \in X \times X^{*}$,

$$
\left(h_{\mathrm{FY}}\right)^{*}\left(x, x^{*}\right)=h_{\mathrm{FY}}\left(x, x^{*}\right) .
$$

Our aim is to prove that any maximal monotone operator has a convex representation with a similar property.

From now on, $T: X \rightrightarrows X^{*}$ is a maximal monotone operator. Define, as in [8], $\mathcal{H}(T)$ to be the family of convex lower semicontinuous functions $h: X \times X^{*} \rightarrow \overline{\mathbb{R}}$ such that

$$
\begin{array}{ll}
\forall\left(x, x^{*}\right) \in X \times X^{*}, \quad h\left(x, x^{*}\right) \geq\left\langle x, x^{*}\right\rangle, \\
\\
\left(x, x^{*}\right) \in T \Rightarrow h\left(x, x^{*}\right)=\left\langle x, x^{*}\right\rangle .
\end{array}
$$

This family is nonempty 8]. Moreover, for any $h \in \mathcal{H}(T), h\left(x, x^{*}\right)=\left\langle x, x^{*}\right\rangle$ if and only if $\left(x, x^{*}\right) \in T$ [7]. Hence, any element of $\mathcal{H}(T)$ fully characterizes, or represents, $T$. Since the sup of convex lower semicontinuous function is also convex and lower semicontinuous, also using (1.2) we conclude that the sup of any (nonempty) subfamily of $\mathcal{H}(T)$ is still in $\mathcal{H}(T)$.

The dual of $X \times X^{*}$ is $X^{*} \times X^{* *}$. So, for $\left(x, x^{*}\right) \in X \times X^{*},\left(y^{*}, y^{* *}\right) \in X^{*} \times X^{* *}$,

$$
\left\langle\left(x, x^{*}\right),\left(y^{*}, y^{* *}\right)\right\rangle=\left\langle x, y^{*}\right\rangle+\left\langle x^{*}, y^{* *}\right\rangle .
$$

Given a function $h: X \times X^{*} \rightarrow \overline{\mathbb{R}}$, define $\mathrm{J} h: X \times X^{*} \rightarrow \overline{\mathbb{R}}$,

$$
\mathcal{J} h\left(x, x^{*}\right):=h^{*}\left(x^{*}, x\right),
$$

where $h^{*}$ stands for the Fenchel-Legendre conjugate of $h$ and the canonical inclusion of $X$ in $X^{* *}$ is being used. Equivalently,

$$
\operatorname{gh}\left(x, x^{*}\right)=\sup _{\left(y, y^{*}\right) \in X \times X^{*}}\left\langle x, y^{*}\right\rangle+\left\langle y, x^{*}\right\rangle-h\left(y, y^{*}\right) .
$$

Trivially, $\mathcal{J}$ inverts the natural order of functions, i.e., if $h \geq h^{\prime}$, then $\mathcal{J} h^{\prime} \geq \mathcal{J} h$. The family $\mathcal{H}(T)$ is invariant under the application $\mathcal{J}[7]$. The aim of this paper is to prove that there exists an element $h \in \mathcal{H}(T)$ such that $\mathcal{J} h=h$.

The application $\mathcal{J}$ can be studied in the framework of generalized conjugation [18] Ch. 11, Sec. L]. With this aim, define

$$
\begin{aligned}
& \Phi:\left(X \times X^{*}\right) \times\left(X \times X^{*}\right): \rightarrow \mathbb{R}, \\
& \Phi\left(\left(x, x^{*}\right),\left(y, y^{*}\right)\right):=\left\langle x, y^{*}\right\rangle+\left\langle y, x^{*}\right\rangle .
\end{aligned}
$$


Given $h: X \times X^{*} \rightarrow \overline{\mathbb{R}}$, let $h^{\Phi}$ be the conjugate of $h$ with respect to the coupling function $\Phi$,

$$
h^{\Phi}\left(x, x^{*}\right):=\sup _{\left(y, y^{*}\right) \in X \times X^{*}} \Phi\left(\left(x, x^{*}\right),\left(y, y^{*}\right)\right)-h\left(y, y^{*}\right) .
$$

Now we have

$$
\mathcal{J} h=h^{\Phi},
$$

and, in particular,

$$
h \geq h^{\Phi \Phi}=\jmath^{2} h .
$$

\section{Proof of the MAIN THEOREM}

Define $\sigma_{T}: X \times X^{*} \rightarrow \overline{\mathbb{R}}$

$$
\sigma_{T}:=\sup _{h \in \mathcal{H}(T)} h .
$$

Since $\mathcal{H}(T)$ is "closed" under the sup operation, we conclude that $\sigma_{T}$ is the biggest element of $\mathcal{H}(T)$. Combining this fact with the inclusion $\mathcal{J} \sigma_{T} \in \mathcal{H}(T)$ we conclude that

$$
\sigma_{T} \geq \partial \sigma_{T}
$$

For a more detailed discussion on $\sigma_{T}$, we refer the reader to [7, eq. (35)]. The above inequality will be, in some sense, our departure point. Now define

$$
\mathcal{H}_{a}(T):=\{h \in \mathcal{H}(T) \mid h \geq \mathcal{J} h\} .
$$

The family $\mathcal{H}_{a}(T)$ is connected with a family of enlargements of $T$ which shares with the $\varepsilon$-subdifferential a special property (see [7]). We already know that $\sigma_{T} \in \mathcal{H}_{a}(T)$. Later on, we will use the following construction of elements in this set.

Proposition 2.1. Take $h \in \mathcal{H}(T)$ and define

$$
\hat{h}=\max h, \mathfrak{J} h .
$$

Then $\hat{h} \in \mathcal{H}_{a}(T)$.

Proof. Since $h$ and $\mathcal{t} h$ are in $\mathcal{H}(T), \hat{h} \in \mathcal{H}(T)$. By definition,

$$
\hat{h} \geq h, \hat{h} \geq \mathcal{J} h .
$$

Applying $\mathcal{J}$ on these inequalities and using (1.6) for majorizing $\mathcal{J}^{2} h$ we obtain

$$
\mathrm{J} h \geq \mathrm{J} \hat{h}, h \geq \mathrm{J} \hat{h} .
$$

Hence, $\hat{h} \geq \mathrm{d} \hat{h}$.

For $h \in \mathcal{H}(T)$ define

$$
L(h):=\{g \in \mathcal{H}(T) \mid h \geq g \geq \mathcal{J} g\} .
$$

The operator $\mathcal{J}$ inverts the order. Therefore, $L(h) \neq \emptyset$ if and only if $h \geq \mathcal{J} h$, i.e., $h \in \mathcal{H}_{a}(T)$. We already know that $L\left(\sigma_{T}\right) \neq \emptyset$.

Proposition 2.2. For any $h \in \mathcal{H}_{a}(T)$, the family $L(h)$ has a minimal element. 
Proof. We shall use the Zorn Lemma. Let $\mathcal{C} \subseteq L(h)$ be a (nonempty) chain, that is, $\mathcal{C}$ is totally ordered. Take $h^{\prime} \in \mathcal{C}$. For any $h^{\prime \prime} \in \mathcal{C}, h^{\prime} \geq h^{\prime \prime}$ or $h^{\prime \prime} \geq h^{\prime}$. In the first case we have $h^{\prime} \geq h^{\prime \prime} \geq \mathcal{J} h^{\prime \prime}$, and in the second case, $h^{\prime} \geq \mathcal{J} h^{\prime} \geq \mathcal{J} h^{\prime \prime}$. Therefore,

$$
h^{\prime} \geq \mathcal{J} h^{\prime \prime}, \forall h^{\prime}, h^{\prime \prime} \in \mathcal{C} .
$$

Now define

$$
\hat{g}=\sup _{h^{\prime} \in \mathcal{C}} \mathcal{J} h^{\prime} .
$$

Since $\mathcal{H}(T)$ is invariant under $\mathcal{J}$ and also closed with respect to the sup, we have $\hat{g} \in \mathcal{H}(T)$. From (2.1), (2.2) it follows that

$$
h^{\prime} \geq \hat{g} \geq \mathcal{J} h^{\prime}, \forall h^{\prime} \in \mathcal{C} .
$$

Applying $\mathcal{J}$ to the above inequalities, and also using (1.6), we conclude that

$$
h^{\prime} \geq \mathcal{J} \hat{g} \geq \mathcal{J} h^{\prime}, \forall h^{\prime} \in \mathcal{C} .
$$

Since $\hat{g} \in \mathcal{H}(T), \partial \hat{g} \in \mathcal{H}(T)$. Taking the sup on $h^{\prime} \in \mathcal{C}$, in the right-hand side of the last inequality, we get

$$
\jmath \hat{g} \geq \hat{g}
$$

Applying $\mathcal{J}$, again, we obtain

$$
\mathcal{\partial} \hat{g} \geq \mathcal{J}(\mathcal{\partial g}) .
$$

Take some $h^{\prime} \in \mathcal{C}$. By the definition of $L(h)$ and (2.3), we conclude that $h \geq h^{\prime} \geq \mathcal{J} \hat{g}$. Hence $\mathcal{J} \hat{g}$ belongs to $L(h)$ and is a lower bound for any element of $\mathcal{C}$. Now we apply the Zorn Lemma to conclude that $L(h)$ has a minimal element.

The minimal elements of $L(h)$ (for $h \in \mathcal{H}_{a}(T)$ ) are the natural candidates for being fixed points of $\mathcal{J}$. First we will show that they are fixed points of $\mathcal{J}^{2}$. Observe that, since $\mathcal{J}$ inverts the order of functions, $\mathcal{J}^{2}$ preserves it, i.e., if $h \geq h^{\prime}$, then $J^{2} h \geq \mathcal{J}^{2} h^{\prime}$. Moreover, $\mathcal{J}^{2}$ maps $\mathcal{H}(T)$ in itself.

Proposition 2.3. Take $h \in \mathcal{H}_{a}(T)$ and let $h_{0}$ be a minimal element of $L(h)$. Then $\mathrm{J}^{2} h_{0}=h_{0}$.

Proof. First observe that $\mathcal{J}^{2} h_{0} \in \mathcal{H}(T)$. By assumption, $h_{0} \geq \mathcal{J} h_{0}$. Applying $\mathcal{J}^{2}$ in this inequality we get

$$
\mathcal{J}^{2} h_{0} \geq \mathcal{J}^{2}\left(\mathcal{J} h_{0}\right)=\mathcal{J}\left(\mathcal{J}^{2} h_{0}\right) .
$$

Since $h \geq h_{0}$ and, by (1.6), $h_{0} \geq \mathcal{J}^{2} h_{0}$, we conclude that $h \geq \mathcal{J}^{2} h_{0} \geq \mathcal{J}\left(\mathcal{J}^{2} h_{0}\right)$. Hence $\mathcal{J}^{2} h_{0} \in L(h)$. Again using the inequality $h_{0} \geq \mathcal{J}^{2} h_{0}$ and the minimality of $h_{0}$, the conclusion follows.

Theorem 2.4. Take $h \in \mathcal{H}(T)$ such that $h \geq \mathcal{J} h$. Then $h_{0} \in L(h)$ is minimal (on $L(h))$ if and only if $h_{0}=\mathcal{J} h_{0}$.

Proof. Assume first that $h_{0}=\mathcal{J} h_{0}$. If $h^{\prime} \in L(h)$ and

$$
h_{0} \geq h^{\prime},
$$

then, applying $\mathcal{J}$ on this inequality and using the definition of $L(h)$ we conclude that

$$
h^{\prime} \geq \mathcal{J} h^{\prime} \geq \mathcal{J} h_{0}=h_{0} .
$$

Combining the above inequalities we obtain $h^{\prime}=h_{0}$. Hence $h_{0}$ is minimal on $L(h)$. 
Assume now that $h_{0}$ is minimal on $L(h)$. By the definition of $L(h), h_{0} \geq \mathcal{J} h_{0}$. Suppose that for some $\left(x_{0}, x_{0}^{*}\right)$,

$$
h_{0}\left(x_{0}, x_{0}^{*}\right)>\mathcal{\partial} h_{0}\left(x_{0}, x_{0}^{*}\right) .
$$

We shall prove that this assumption is contradictory. By Proposition 2.3, $h_{0}=$ $\mathcal{J}\left(\mathcal{J} h_{0}\right)$. Hence, the above inequality can be expressed as

$$
\mathcal{J}\left(\mathcal{J} h_{0}\right)\left(x_{0}, x_{0}^{*}\right)>\mathcal{J} h_{0}\left(x_{0}, x_{0}^{*}\right),
$$

or equivalently

$$
\sup _{\left(y, y^{*}\right) \in X \times X^{*}}\left\langle y, x_{0}^{*}\right\rangle+\left\langle x_{0}, y^{*}\right\rangle-\mathcal{J} h_{0}\left(y, y^{*}\right)>\mathcal{J} h_{0}\left(x_{0}, x_{0}^{*}\right) .
$$

Therefore, there exists some $\left(y_{0}, y_{0}^{*}\right) \in X \times X^{*}$ such that

$$
\left\langle y_{0}, x_{0}^{*}\right\rangle+\left\langle x_{0}, y_{0}^{*}\right\rangle-\partial h_{0}\left(y_{0}, y_{0}^{*}\right)>\mathcal{\partial} h_{0}\left(x_{0}, x_{0}^{*}\right) .
$$

In particular, $\mathcal{\partial} h_{0}\left(y_{0}, y_{0}^{*}\right), \mathcal{\partial} h_{0}\left(x_{0}, x_{0}^{*}\right) \in \mathbb{R}$. Interchanging $\mathcal{\partial} h_{0}\left(y_{0}, y_{0}^{*}\right)$ with $\mathcal{\partial} h_{0}\left(x_{0}, x_{0}^{*}\right)$ we get

$$
\left\langle y_{0}, x_{0}^{*}\right\rangle+\left\langle x_{0}, y_{0}^{*}\right\rangle-\mathcal{J} h_{0}\left(x_{0}, x_{0}^{*}\right)>\partial h_{0}\left(y_{0}, y_{0}^{*}\right) .
$$

Therefore, also using (1.4), we get $\mathcal{J}\left(\mathcal{J} h_{0}\left(y_{0}, y_{0}^{*}\right)\right)>\mathcal{J} h_{0}\left(y_{0}, y_{0}^{*}\right)$. Again using the equality $\mathcal{J}^{2} h_{0}=h_{0}$ we conclude that

$$
h_{0}\left(y_{0}, y_{0}^{*}\right)>\mathcal{\partial} h_{0}\left(y_{0}, y_{0}^{*}\right) .
$$

Define $\gamma: X \times X^{*} \rightarrow \mathbb{R}, g: X \times X^{*} \rightarrow \overline{\mathbb{R}}$

$$
\begin{aligned}
\gamma\left(x, x^{*}\right) & :=\left\langle x, y_{0}^{*}\right\rangle+\left\langle y_{0}, x^{*}\right\rangle-\partial h_{0}\left(y_{0}, y_{0}^{*}\right), \\
g & :=\max \gamma, \partial h_{0} .
\end{aligned}
$$

By (1.4), $h_{0} \geq \gamma$. Since $h_{0} \in L(h), h_{0} \geq \mathcal{J} h_{0}$. Therefore,

$$
h_{0} \geq g \geq \mathcal{J} h_{0} .
$$

We claim that $g \in \mathcal{H}(T)$. Indeed, $g$ is a lower semicontinuous convex function. Moreover, since $h_{0}, \mathcal{J} h_{0} \in \mathcal{H}(T)$, it follows from (1.2) and the above inequalities that $g \in \mathcal{H}(T)$. Now apply $\mathcal{J}$ to the above inequality to conclude that

$$
h_{0} \geq \mathcal{J} g \geq \mathcal{J} h_{0} .
$$

Therefore, defining

$$
\hat{g}=\max g, \partial g,
$$

we have $h>h_{0} \geq \hat{g}$. By Proposition 2.1 $\hat{g} \in \mathcal{H}(T)$ and $\hat{g} \geq \mathcal{J} \hat{g}$. Combining these results with the minimality of $h_{0}$, it follows that $\hat{g}=h_{0}$. In particular,

$$
\hat{g}\left(y_{0}, y_{0}^{*}\right)=h_{0}\left(y_{0}, y_{0}^{*}\right) \text {. }
$$

To conclude the proof we shall evaluate $\hat{g}\left(y_{0}, y_{0}^{*}\right)$. Using (2.7) we obtain

$$
\gamma\left(y_{0}, y_{0}^{*}\right)=2\left\langle y_{0}, y_{0}^{*}\right\rangle-\partial h_{0}\left(y_{0}, y_{0}^{*}\right) .
$$

Since $\mathcal{J} h_{0} \in \mathcal{H}(T), \mathcal{J} h_{0}\left(y_{0}, y_{0}^{*}\right) \geq\left\langle y_{0}, y_{0}^{*}\right\rangle$. Hence, $\gamma\left(y_{0}, y_{0}^{*}\right) \leq\left\langle y_{0}, y_{0}^{*}\right\rangle$ and by (2.8)

$$
g\left(y_{0}, y_{0}^{*}\right)=\partial h_{0}\left(y, y^{*}\right) .
$$

Again using the inequality $g \geq \gamma$, we have

$$
\mathcal{\partial} \gamma\left(y_{0}, y_{0}^{*}\right) \geq \mathcal{J g}\left(y_{0}, y_{0}^{*}\right) \text {. }
$$

Direct calculation yields $\mathcal{J} \gamma\left(y_{0}, y_{0}^{*}\right)=\mathcal{J} h_{0}\left(y, y^{*}\right)$. Therefore

$$
\partial h_{0}\left(y_{0}, y_{0}^{*}\right) \geq \mathcal{J} g\left(y_{0}, y_{0}^{*}\right) \text {. }
$$


Combining (2.11), (2.12) and (2.9) we obtain

$$
\hat{g}\left(y_{0}, y_{0}^{*}\right)=\mathcal{J} h_{0}\left(y_{0}, y_{0}^{*}\right) \text {. }
$$

This equality, together with (2.10), yields $h_{0}\left(y_{0}, y_{0}^{*}\right)=\mathcal{J} h_{0}\left(y_{0}, y_{0}^{*}\right)$, in contradiction with (2.6). Therefore, $h_{0}\left(x, x^{*}\right)=\mathcal{J} h_{0}\left(x, x^{*}\right)$ for all $\left(x, x^{*}\right)$.

Since $\sigma_{T} \in \mathcal{H}_{a}(T), L\left(\sigma_{T}\right) \neq \emptyset$ and there exists some $h \in L\left(\sigma_{T}\right)$ such that $\mathcal{J} h=h$. (Indeed $L\left(\sigma_{T}\right)=\mathcal{H}_{a}(T)$.)

\section{Application}

Let $f: X \rightrightarrows X^{*}$ be a proper lower semicontinuous convex function. We already know that $\partial f$ is maximal monotone. Define, for $\varepsilon \geq 0$,

$$
\partial_{\varepsilon} f(x):=\left\{x^{*} \in X^{*} \mid f(y) \geq f(x)+\left\langle y-x, x^{*}\right\rangle-\varepsilon, \forall y \in X\right\} .
$$

Note that $\partial_{0} f=\partial f$. We also have

$$
\begin{aligned}
& \partial f(x) \subseteq \partial_{\varepsilon} f(x), \forall x \in X, \varepsilon \geq 0, \\
& 0 \leq \varepsilon_{1} \leq \varepsilon_{2} \Rightarrow \partial_{\varepsilon_{1}} f(x) \subseteq \partial_{\varepsilon_{2}} f(x), \forall x \in X .
\end{aligned}
$$

Property (3.1) tells that $\partial_{\varepsilon} f$ enlarges $\partial f$. Property (3.2) shows that $\partial_{\varepsilon} f$ is nondecreasing (or increasing) in $\varepsilon$. The operator $\partial_{\varepsilon} f$ has been introduced in [3], and since that, it has had may theoretical and algorithmic applications [1, 14, 9, 10, 22, 12, 2].

Since $\partial f$ is maximal monotone, the enlarged operator $\partial_{\varepsilon} f$ loses monotonicity in general. Even though, we have

$$
x^{*} \in \partial_{\varepsilon} f(x) \Rightarrow\left\langle x-y, x^{*}-y^{*}\right\rangle \geq-\varepsilon, \forall\left(y, y^{*}\right) \in \partial f .
$$

Now, take

$$
\begin{aligned}
& x_{1}^{*} \in \partial_{\varepsilon_{1}} f\left(x_{1}\right), x_{2}^{*} \in \partial_{\varepsilon_{1}} f\left(x_{2}\right), \\
& p, q \geq 0, p+q=1,
\end{aligned}
$$

and define

$$
\begin{aligned}
& \left(\bar{x}, \bar{x}^{*}\right):=p\left(x_{1}, x_{1}^{*}\right)+q\left(x_{2}, x_{2}^{*}\right), \\
& \bar{\varepsilon}:=p \varepsilon_{1}+q \varepsilon_{2}+p q\left\langle x_{1}-x_{2}, x_{1}^{*}-x_{2}^{*}\right\rangle .
\end{aligned}
$$

Using the previous definitions, and the convexity of $f$, it is trivial to check that

$$
\bar{\varepsilon} \geq 0, \bar{x}^{*} \in \partial_{\bar{\varepsilon}} f(\bar{x}) .
$$

Properties (3.4), (3.5), (3.6) will be called a transportation formula. If $\varepsilon_{1}=\varepsilon_{2}=0$, then we are using elements in the graph of $\partial f$ to construct elements in the graph of $\partial_{\varepsilon} f$. In (3.5), the product of elements in $\partial_{\varepsilon} f$ appears. This product admits the following estimation:

$$
x_{1}^{*} \in \partial_{\varepsilon_{1}} f\left(x_{1}\right), x_{2}^{*} \in \partial_{\varepsilon_{1}} f\left(x_{2}\right) \Rightarrow\left\langle x_{1}-x_{2}, x_{1}^{*}-x_{2}^{*}\right\rangle \geq-\left(\varepsilon_{1}+\varepsilon_{2}\right) .
$$

Moreover, $\partial_{\varepsilon} f$ is maximal with respect to property (3.7). We will call property (3.7) additivity. The enlargement $\partial_{\varepsilon} f$ can be characterized by the function $h_{\mathrm{FY}}$, defined in (1.1),

$$
x^{*} \in \partial_{\varepsilon} f(x) \Longleftrightarrow h_{\mathrm{FY}}\left(x, x^{*}\right) \leq\left\langle x, x^{*}\right\rangle+\varepsilon .
$$

The transportation formula (3.4), (3.5), (3.6) now follows directly of the convexity of $h_{\mathrm{FY}}$. Additivity follows from the fact that $h_{\mathrm{FY}} \geq \mathcal{J} h_{\mathrm{FY}}$, and maximality of the additivity follows from the fact that

$$
h_{\mathrm{FY}}=\mathcal{J} h_{\mathrm{FY}} .
$$


Define the graph of $\partial_{\varepsilon} f$ as

$$
G\left(\partial_{(\cdot)} f(\cdot)\right):=\left\{\left(x, x^{*}, \varepsilon\right) \mid x^{*} \in \partial_{\varepsilon} f(x)\right\} .
$$

Note that $G\left(\partial_{(\cdot)} f(\cdot)\right)$ is closed. So we say that $\partial_{\varepsilon} f$ is closed.

Given $T: X \rightrightarrows X^{*}$, maximal monotone, it would be desirable to have an enlargement of $T$, say $T^{\varepsilon}$, with similar properties to the $\partial_{\varepsilon} f$ enlargement of $\partial f$. With this aim, such an object was defined in [4, 5] (in finite-dimensional spaces and in Banach spaces, respectively), for $\varepsilon \geq 0$, as

$$
T^{\varepsilon}(x):=\left\{x^{*} \in X^{*} \mid\left\langle x-y, x^{*}-y^{*}\right\rangle \geq-\varepsilon, \forall\left(y, y^{*}\right) \in T\right\} .
$$

The $T^{\varepsilon}$ enlargement of $T$ shares many properties with the $\partial_{\varepsilon} f$ enlargement of $\partial f$ : the transportation formula, Lipschitz continuity (in the interior of its domain), and even the Brøndsted-Rockafellar property (in Reflexive Banach spaces). Since its introduction, it has had both theoretical and algorithmic applications [4, 6, 20, 21, 15. 16]. Even though, $T^{\varepsilon}$ is not the extension of the construct $\partial_{\varepsilon} f$ to a generic maximal monotone operator. Indeed, taking $T=\partial f$, we obtain

$$
\partial_{\varepsilon} f(x) \subseteq(\partial f)^{\varepsilon}(x)
$$

with examples of strict inclusion even in finite-dimensional cases [4]. Therefore, in general, $T^{\varepsilon}$ lacks the "additive" property (3.7). The $T^{\varepsilon}$ enlargement satisfies a weaker property [5]

$$
x_{1}^{*} \in T^{\varepsilon_{1}}\left(x_{1}\right), x_{2}^{*} \in T^{\varepsilon_{2}}\left(x_{2}\right) \Rightarrow\left\langle x_{1}-x_{2}, x_{1}^{*}-x_{2}^{*}\right\rangle \geq-\left(\sqrt{\varepsilon_{1}}+\sqrt{\varepsilon_{2}}\right)^{2} .
$$

The enlargement $T^{\varepsilon}$ is also connected with a convex function. Indeed,

$$
\begin{aligned}
x^{*} \in T^{\varepsilon}(x) & \Longleftrightarrow\left\langle x-y, x^{*}-y^{*}\right\rangle \geq-\varepsilon, \forall\left(y, y^{*}\right) \in T \\
& \Longleftrightarrow \sup _{\left(y, y^{*}\right) \in T}\left\langle x-y, y^{*}-x\right\rangle \leq \varepsilon .
\end{aligned}
$$

The Fitzpatrick function, $\varphi_{T}$, is the smallest element of $\mathcal{H}(T)$ [ $]$, and is defined as

$$
\varphi_{T}\left(x, x^{*}\right):=\sup _{\left(y, y^{*}\right) \in T}\left\langle x-y, y^{*}-x\right\rangle+\left\langle x, x^{*}\right\rangle .
$$

Therefore,

$$
x^{*} \in T^{\varepsilon}(x) \Longleftrightarrow \varphi_{T}\left(x, x^{*}\right) \leq\left\langle x, x^{*}\right\rangle+\varepsilon .
$$

Now, the transportation formula for $T^{\varepsilon}$ follows from convexity of $\varphi_{T}$. In [7] it is proven that each enlargement $\hat{T}^{\varepsilon}$ of $T$, which has a closed graph, is nondecreasing and satisfies the transportation formula, is characterized by a function $\hat{h} \in \mathcal{H}(T)$, by the formula

$$
x^{*} \in \hat{T}^{\varepsilon}(x) \Longleftrightarrow \hat{h}\left(x, x^{*}\right) \leq\left\langle x, x^{*}\right\rangle+\varepsilon .
$$

So, if we want to retain "additivity",

$$
x_{1}^{*} \in \hat{T}^{\varepsilon_{1}}\left(x_{1}\right), x_{2}^{*} \in \hat{T}^{\varepsilon_{2}}\left(x_{2}\right) \Rightarrow\left\langle x_{1}-x_{2}, x_{1}^{*}-x_{2}^{*}\right\rangle \geq-\left(\varepsilon_{1}+\varepsilon_{2}\right) .
$$

We shall require $\hat{h} \geq \mathcal{\partial} \hat{h}$. The enlargements in this family, which are also maximal with respect to the additivity, are structurally closer to the $\partial_{\varepsilon} f$ enlargement, and are characterized by $\hat{h} \in \mathcal{H}(T)$,

$$
\hat{h}=\partial \hat{h} .
$$

If there were only one element in $\mathcal{H}(T)$ as the fixed point of $\mathcal{J}$, then this element would be the "canonical" representation of $T$ by a convex function, and the associated enlargement would be the extension of the $\varepsilon$-subdifferential enlargement to $T$. Unfortunately, it is not clear whether we have uniqueness of such fixed points. 
Existence of an additive enlargement of $T$, maximal with respect to "additivity", was proved in [23]. The convex representation of this enlargement turned out to be minimal in the family $\mathcal{H}_{a}(T)$, but the characterization of these minimal elements of $\mathcal{H}_{a}(T)$ as fixed point of $\mathcal{J}$ was lacking.

Since the function $\sigma_{T}$ has played a fundamental role in our proof, we redescribe it here. Let $\delta_{T}$ be the indicator function of $T$, i.e., in $T$ its value is 0 and elsewhere in $\left(X \times X^{*} \backslash T\right)$ its value is $+\infty$. Denote the duality product by $\pi: X \times X^{*} \rightarrow \mathbb{R}$, $\pi\left(x, x^{*}\right)=\left\langle x, x^{*}\right\rangle$. Then

$$
\sigma_{T}\left(x, x^{*}\right)=\mathrm{cl}-\operatorname{conv}\left(\pi+\delta_{T}\right),
$$

where $\mathrm{cl}-\operatorname{conv} f$ stands for the biggest lower semicontinuous convex function majorized by $f$. We refer the reader to 7$]$ for a detailed analysis of this function.

\section{ACKNOWLEDGEMENT}

We thank the anonymous referee for the suggestions which helped to improve this paper.

\section{REFERENCES}

1. D.P. Bertsekas and S.K. Mitter. A descent numerical method for optimization problems with nondifferentiable cost functionals. SIAM Journal on Control, 11(4):637-652, 1973. MR 48:7592

2. F. Bonnans, J.Ch. Gilbert, C.L. Lemaréchal and C.A. Sagastizábal. Optimisation Numérique, aspects théoriques et pratiques. Collection "Mathématiques et applications", SMAI-SpringerVerlag, Berlin, 1997. MR 99f:90001

3. A. Brøndsted and R. T. Rockafellar. On the subdifferentiability of convex functions. Proceedings of the American Mathematical Society, 16:605-611, 1965. MR 31:2361

4. Regina S. Burachik, Alfredo N. Iusem, and B. F. Svaiter. Enlargement of monotone operators with applications to variational inequalities. Set-Valued Analysis, 5(2):159-180, 1997. MR 98h:49010

5. Regina Sandra Burachik and B. F. Svaiter. $\varepsilon$-enlargements of maximal monotone operators in Banach spaces. Set-Valued Analysis, 7(2):117-132, 1999. MR 2000i:47099

6. Regina S. Burachik, Claudia A. Sagastizábal, and B. F. Svaiter. $\epsilon$-enlargements of maximal monotone operators: theory and applications. In M. Fukushima and L. Qi, editors, Reformulation: nonsmooth, piecewise smooth, semismooth and smoothing methods (Lausanne, 1997), volume 22 of Applied Optimization, pages 25-43. Kluwer Acad. Publ., Dordrecht, 1999. MR 2000a: 49030

7. Burachik, R.S. and Svaiter, B.F.: Maximal monotone operators, convex functions and a special family of enlargements, Set Valued Analysis, (to appear).

8. Fitzpatrick, S.: Representing monotone operators by convex functions, Workshop/Miniconference on Functional Analysis and Optimization (Canberra, 1988) 59-65, Proc. Centre Math. Anal. Austral. Nat. Univ., 20 Austral. Nat. Univ., Canberra, 1988. MR 90i: 47054

9. J.-B. Hiriart-Urruty and C. Lemaréchal. Convex Analysis and Minimization Algorithms. Number 305-306 in Grund. der math. Wiss. Springer-Verlag, 1993. (two volumes). MR 95m:90001; MR 95m:90002

10. K.C. Kiwiel. Proximity control in bundle methods for convex nondifferentiable minimization. Mathematical Programming, 46:105-122, 1990. MR 91c:90084

11. Krauss, Eckehard: A representation of maximal monotone operators by saddle functions, Rev. Roumaine Math. Pures Appl. 30 (1985), 823-837. MR 87e:47074

12. C. Lemaréchal, A. Nemirovskii, and Yu. Nesterov. New variants of bundle methods. Mathematical Programming, 69:111-148, 1995. MR 96g:90075

13. Martinez-Legaz, J.-E. and Théra, M.: A convex representation of maximal monotone operators, Journal of Nonlinear and Convex Analysis 2 (2001), 243-247. MR 2002e:49035 
14. E.A. Nurminski. $\varepsilon$-subgradient mapping and the problem of convex optimization. Cybernetics, 21(6):796-800, 1986.

15. Revalski, J.P. and M. Théra, Generalized sums of monotone operators, C. R. Acad. Sci. Paris Sér. I Math. 329(11):979-984, 1999. MR 2000m:47068

16. Revalski, J.P. and M. Théra, Variational and extended sums of monotone operators, Ill-posed variational problems and regularization techniques (Trier, 1998), 229-246, Lecture Notes in Econom. and Math. Systems, 477, Springer, Berlin, 1999. MR 2000k:47071

17. Rockafellar, R. T.: On the maximal monotonicity of subdifferential mappings, Pacific Journal of Mathematics 33 (1970), 209-216. MR 41:7432

18. R. Tyrrell Rockafellar, Roger J-B. Wets. Variational Analysis. Springer Verlag, Berlin Heidelberg, 1998. MR 98m:49001

19. Simons, S.: Minimax and monotonicity. Lecture Notes in Mathematics, 1693. Springer-Verlag, Berlin, 1998. MR 2001h:49002

20. M. V. Solodov and B. F. Svaiter. An inexact hybrid extragradient-proximal point algorithm using the enlargement of a maximal monotone operator. Set-Valued Analysis, 7(4):323-345, December 1999. MR 2001a:90084

21. M. V. Solodov and B. F. Svaiter. Error bounds for proximal point subproblems and associated inexact proximal point algorithms. Mathematical Programming, 88(2):371-389, 2000. MR 2001f: 90052

22. H. Schramm and J. Zowe. A version of the bundle idea for minimizing a nonsmooth function: conceptual idea, convergence analysis, numerical results. SIAM Journal on Optimization, 2(1):121-152, 1992. MR 93b:90089

23. B. F. Svaiter. A Family of Enlargements of Maximal Monotone Operators. Set-Valued Analysis, 8(4):311-328, December 2000. MR 2001i:49037

impa instituto de Matemática Pura e Aplicada, Estrada Dona Castorina 110, Rio DE JANEIRO-RJ, CEP 22460-320 BRAZIL

E-mail address: benar@impa.br 